\title{
Association between Speech Recognition in Noise and Risk Factors of Cardiovascular Disease
}

\author{
Thadé Goderie $^{\mathrm{a}}$ Marieke F. van Wier ${ }^{\mathrm{a}}$ Mariska Stam $^{\mathrm{a}}$ \\ Birgit I. Lissenberg-Witte ${ }^{b}$ Paul Merkus $^{\text {a }}$ Cas Smits $^{\text {a }}$ Sophia E. Kramer ${ }^{a}$ \\ aDepartment of Otolaryngology-Head and Neck surgery, Section Ear and Hearing, Amsterdam Public Health \\ Research Institute, Amsterdam UMC, Vrije Universiteit Amsterdam, Amsterdam, The Netherlands; ${ }^{b}$ Department of \\ Epidemiology and Data Science, Amsterdam UMC, Vrije Universiteit Amsterdam, Amsterdam, The Netherlands
}

\author{
Keywords \\ Cardiovascular disease - Epidemiology · Hearing loss . \\ Longitudinal research · Presbycusis · Risk factors · \\ Comorbidity
}

\begin{abstract}
Introduction: Risk factors for cardiovascular disease (CVD) are associated with sensorineural hearing loss. CVD risk factors are known to cluster and interact, thereby increasing the cumulative risk for CVD. Previously, using the database of the Netherlands Longitudinal Study on Hearing (NL-SH), an association was found between a history of smoking and an increased decline in speech recognition in noise over 10 years of follow-up. Prospectively limited data are available on the association between CVD risk factors, interactions of these risk factors, and hearing loss. In this study, data from the NL-SH were used to study the association between CVD risk factors and speech recognition in noise longitudinally. Methods: Baseline, 5-year, and 10-year follow-up data of the $\mathrm{NL}-\mathrm{SH}$ were included. The NL-SH is a web-based prospective cohort study which started in 2006. Participants were aged 18-70 years at baseline. Speech recognition in noise was determined with an online digit-triplet speech-in-noise test. In
\end{abstract}

addition, participants completed online questionnaires on demographic, lifestyle, and health-related characteristics. The association of the ability to recognize speech in noise with CVD risk factors (i.e., obesity, rheumatoid arthritis [RA], hypertension, diabetes mellitus, and dyslipidemia) was analyzed longitudinally. We also analyzed the interaction between these risk factors (including age, sex, and history of smoking) and speech recognition in noise. Results: None of the CVD risk factors or interactions of 2 CVD risk factors was significantly associated with a decline in SRT over time. Obesity $(p=0.016), \operatorname{RA}(p=0.027)$, and hypertension $(p=0.044)$ were associated with overall higher (more unfavorable) SRTs. No overall interactions between CVD risk factors were found. Conclusion: Obesity, RA, and hypertension were overall associated with a higher SRT, but no longitudinal associations between these or other CVD factors with SRTs were found. Also, no interactions between 2 CVD risk factors and SRTs were found. Although no longitudinal associations between CVD risk factors and decline in SRTs were found, clinicians should be alert about the concurrent association between CVD risk factors and hearing loss.

C 2021 The Author(s)

Published by S. Karger AG, Basel karger@karger.com www.karger.com/aud

Karger $\stackrel{\text { ' }}{5}$

BOPEN ACCESS
(C) 2021 The Author(s)

Published by S. Karger AG, Basel

This is an Open Access article licensed under the Creative Commons Attribution-NonCommercial-4.0 International License (CC BY-NC) (http://www.karger.com/Services/OpenAccessLicense), applicable to the online version of the article only. Usage and distribution for commercial purposes requires written permission.
Thadé Goderie

Department of Otolaryngology - Head and Neck surgery, Section Ear \& Hearing, Amsterdam Public Health Research Institute, Amsterdam UMC, Vrije Universiteit Amsterdam, De Boelelaan 1117, NL-1081 HV Amsterdam (The Netherlands) t.goderie@amsterdamumc.nl 


\section{Introduction}

Hearing loss is one of the most frequently occurring chronic conditions worldwide [Vos et al., 2016]. Hearing loss has been associated with cardiovascular disease (CVD) and risk factors for CVD [Oron et al., 2014; Besser et al., 2018]. Evidence shows that the major risk factors for CVD are sex, age, dyslipidemia, tobacco smoking, hypertension, obesity, rheumatoid arthritis (RA), and diabetes mellitus (DM) [D'Agostino et al., 2008; Choy et al., 2014; Lu et al., 2014]. Risk factors for CVD cluster and interact, increasing the cumulative risk of a cardiovascular event [Anderson et al., 1991; D’Agostino et al., 2008]. This may also be the case for the risk of developing hearing loss.

In a previous study using the Netherlands Longitudinal Study on Hearing (NL-SH), we found that a history of tobacco smoking was related to an increased rate of decline in speech recognition in noise [Goderie et al., 2020]. The mechanism to explain this association is unclear, but it is hypothesized that atherosclerosis, the major cause of CVD, plays a role in the association [Makishima, 1978; Guo et al., 2005; Nomiya et al., 2008]. The cochlea could be susceptible to ischemic damage caused by atherosclerosis because it is an end organ, with a high energy metabolism, dependent on terminal circulation without anastomosis to compensate for decreased blood flow [Kim and Lee, 2009]. Therefore, it can be hypothesized that other CVD risk factors than tobacco smoking that are implicated with atherosclerosis could also affect hearing.

Several large-population studies found an association between the major risk factors for CVD and hearing loss [Agrawal et al., 2009; Shargorodsky et al., 2010; Cruickshanks et al., 2015; Huang et al., 2018; Tan et al., 2018]. Tan et al. [2018] found a graded association between hearing loss and overall cardiovascular risk by using the Framingham 10-year risk score [Tan et al., 2018]. Others did not find (some of) these associations [Shargorodsky et al., 2010; Lin et al., 2011]. Lin et al. [2011] argued that these inconsistent results are possibly because of the weak association between CVD risk factors and hearing loss and because other stronger risk factors may obscure them, such as age. Differences in the design of the studies could also explain the conflicting results. Only a few studies published to date have used a longitudinal design or examined interactions between risk factors for CVD and hearing ability [Linssen et al., 2014; Cruickshanks et al., 2015; Rigters et al., 2018]. A longitudinal study design has the advantage that it allows examination of the individu- al change (in hearing) over time and identification of factors possibly associated with that change [Morrell et al., 2009].

The aim of this study was to analyze the longitudinal (10-year) association between CVD risk factors (i.e., DM, hypertension, dyslipidemia, obesity, and RA) and change of hearing status in a cohort of adults with varying levels of hearing ability, ranging from normal to poor hearing. We also investigated interaction effects between these risk factors and change of hearing status. In addition, we analyzed the overall effect of these CVD risk factors and interactions between these risk factors on hearing status.

Age, sex, and history of smoking were included in the models as potential confounders or moderators. Each of these variables has been examined as single risk factors for hearing loss in a previous study using the NL-SH cohort [Goderie et al., 2020].

\section{Materials and Methods}

\section{Study Design and Settings}

We used data of the Netherlands Longitudinal Study on Hearing (NL-SH). The NL-SH is an ongoing prospective cohort study which is conducted online. It uses a convenience sampling method. The NL-SH includes adults who were aged $18-70$ years at study entry. After the inclusion measurements, follow-up measurements are done every 5 years. Baseline data collection (T0) started in 2006 and is still open for inclusion. The second measurement round (T1) started in 2011, and the third one (T2) started in 2016. Further details about participant recruitment, data collection, and followup measurement rounds have been reported by Stam et al. [2015]. The survey combines a questionnaire with a speech-in-noise test known as the national hearing test (NHT) [Smits et al., 2006] to measure each participant's hearing status. The NHT measures the ability to understand digit triplets in noise by determining the signal-to-noise ratio (SNR) that corresponds to $50 \%$ intelligibility (i.e., the speech reception threshold [SRT] in dB SNR) [Smits et al., 2004]. The NHT yields a score that was categorized (for descriptive purposes only) as good hearing (SRT $<-5.5 \mathrm{~dB}$ SNR), insufficient hearing $(-5.5 \mathrm{~dB}$ SNR $\leq$ SRT $\leq-2.8 \mathrm{~dB}$ SNR), or poor hearing (SRT $>-2.8 \mathrm{~dB}$ SNR) [Smits et al., 2006]. Thus, higher SRTs represent poorer speech recognition abilities in noise, and lower SRTs represent better speech recognition abilities in noise. The NL-SH study protocol was approved by the Medical Ethics Committee of the Amsterdam UMC, location VUmc in Amsterdam, the Netherlands.

Dependent Variable: Speech Recognition Ability in Noise

Participants performed the NHT at each measurement round (T0, T1, and T2) and were advised to use headphones, but could also use loudspeakers. They had to indicate which transducer type (headphones or speakers) they had used during testing and whether they had used a hearing aid during the test. Participants were instructed to perform the test in a quiet room. In addition, participants were asked to rate their hearing ability on a nine-point Likert scale. The 
question asked was "Please rate your hearing when you are not wearing hearing aids ( 1 = very poor and $9=$ very good)." Only unaided scores (tests performed without a hearing aid) were used for the analyses. Participants with an SRT of $\geq 3.0 \mathrm{~dB}$ SNR at the first included measurement round (either T0 or T1) were excluded from the analyses as these SRTs are in the ceiling range of the test $(4.0 \mathrm{~dB}$ SNR), and therefore further decline cannot be measured. Participants who had a change in SRT of over 2 standard deviations (SD) between 2 measurement rounds were excluded when this change was not supported by a change in how participants rated their hearing ability (defined as $<2$-point change on the nine-point scale).

\section{Independent Variables}

Demographic and health-related information was collected through a set of online questionnaires. Age at baseline was categorized into 5 age groups: $18-30,31-40,41-50,51-60$, and $61-70$ years. Questionnaire responses on self-reported chronic medical conditions and self-reported medication use were used to compile the participant's medical information and determine if a chronic condition was present. Self-rated presence of chronic medical conditions was assessed by questions based on the 1989-1990 health survey of Statistics Netherlands [Mootz and Van Den Berg, 1989]. Participants were asked to tick the box for a chronic condition if it was present now or had been during the previous 12 months. This list included 27 conditions, but for the current study, only the presence of "inflammation of the joints of hands and/or feet (RA)," $\mathrm{DM}$, and hypertension was included.

Medication use was also assessed. This was done with the questionnaire "Treatment Inventory of Costs in patients with psychiatric disorders" [Bouwmans et al., 2013], which asks for names of medication used in the past 4 weeks, excluding medication used during hospital stay or contraceptives. A maximum of 10 medications could be reported.

If participants used medication for DM or hypertension (except for $\beta$-blockers as these are commonly used for other conditions as well), they were considered having these diseases, even if they had not reported the presence of that disease in the self-report list of chronic conditions. Because dyslipidemia was not present in the list of chronic conditions and because blood results were not available, we considered participants to have dyslipidemia when they used lipid modifying agents, that is, statins.

At T2, participants are asked to report their height and current body weight. Their BMI was calculated by dividing their weight in kilograms by their squared height in meters. Obesity was defined as a BMI $\geq 30$ [Kopelman, 2000] and analyzed as a dichotomous variable (obese or not obese). We used this variable as a time-independent variable, and thus obese participants at T2 were considered to be obese also at T0 and T1. BMI is known to be fairly stable over time in individuals [Katzmarzyk et al., 1999; Heo et al., 2002].

Tobacco smoking was measured by asking participants if and how often they smoked. Answer categories were "yes, I smoke every day," "yes, I smoke occasionally," "no, but I used to smoke every day," "no, but I used to smoke occasionally," and "no, I never smoked." Participants who had chosen the option "no, I never smoked" were considered having no history of tobacco smoking. All others were categorized as having a history of tobacco smoking. History of tobacco smoking was entered to the model as a time-dependent variable.

As sex, history of tobacco smoking, and age have been investigated as risk factors for hearing loss in our previous study using the NL-SH data [Goderie et al., 2020], these factors were in the current study only included as moderators (i.e., for interactions with other CVD risk factors) or as confounders.

\section{Study Sample}

People aged 18-70 years at baseline who took part in the NL$\mathrm{SH}$ and who had completed a T1 and/or T2 measurement before March 1, 2018, were included in this study. As we evaluated longitudinal data, participants eligible for analyses were required to have completed a minimum of 2 measurement rounds after applying all exclusion criteria, that is, at least T0 and T1, T0 and T2, or T1 and T2. Participants were asked "What was the onset of your hearing loss? (1) Gradual, (2) sudden, or (3) congenital." Participants who consistently reported a congenital hearing loss (i.e., at all measurement rounds) were excluded from the analyses as well as participants who received a cochlear implant during the study or prior to study entry.

\section{Statistical Analysis: Descriptive Analysis}

Means and SDs were calculated for continuous variables. Percentages were calculated for categorical variables.

\section{Statistical Analysis: Differences in Change in SRT over Time}

A linear mixed model was built to examine the longitudinal association of CVD risk factors and SRT. The maximum likelihood method was chosen with a compound symmetry covariance structure for the repeated measurements within participants. The base model included a random intercept for each participant, transducer type (headphones or loudspeakers), and time (T0, T1, and T2) (i.e., base model $=$ random intercept + transducer type + time $)$. Transducer type was included because, on average, better SRTs are measured in the NHT when using headphones compared to speakers [Smits et al., 2006]. A likelihood ratio test was used to analyze if including a random slope significantly improved the model. A random slope accounts for individual variation in the change of the SRT over time. First, the longitudinal associations between risk factors of CVD and SRT were tested in the base model by adding interactions of each risk factor with time (e.g., base model + hypertension + time + hypertension $\times$ time). Variables tested for an interaction with time were dyslipidemia, hypertension, DM, obesity, and RA. Subsequently, risk factors were combined to analyze the possibility of a 3 -way interaction between 2 risk factors and time (e.g., base model $+\mathrm{DM}+$ hypertension + time $+\mathrm{DM} \times$ hypertension $+\mathrm{DM} \times$ time + hypertension $\times$ time $+\mathrm{DM} \times$ hypertension $\times$ time). In addition, sex, history of smoking, and age group were analyzed in the 3 -way interactions. Estimated marginal means (EMMs) were calculated. Only interactions with a minimum of 15 participants per subgroup were analyzed. In the example of the interaction between DM, hypertension, and time, this means that in all 3 measurement rounds, at least 15 participants had to be exposed to both DM and hypertension, 15 participants had to be exposed to DM but not hypertension, 15 participants had to be exposed to hypertension but not DM, and 15 participants had to have no DM and no hypertension. This was done to avoid drawing conclusions on a sample with low power. Unadjusted models and models adjusted for confounders were calculated for the included independent variables. A variable was considered a relevant confounder and included in the model if (1) the potential confounder had significant influence $(p<0.05)$ on the outcome variable and (2) when the regression coefficient of the interaction of interest changed $\geq 10 \%$ after adding the potential confounder to the 


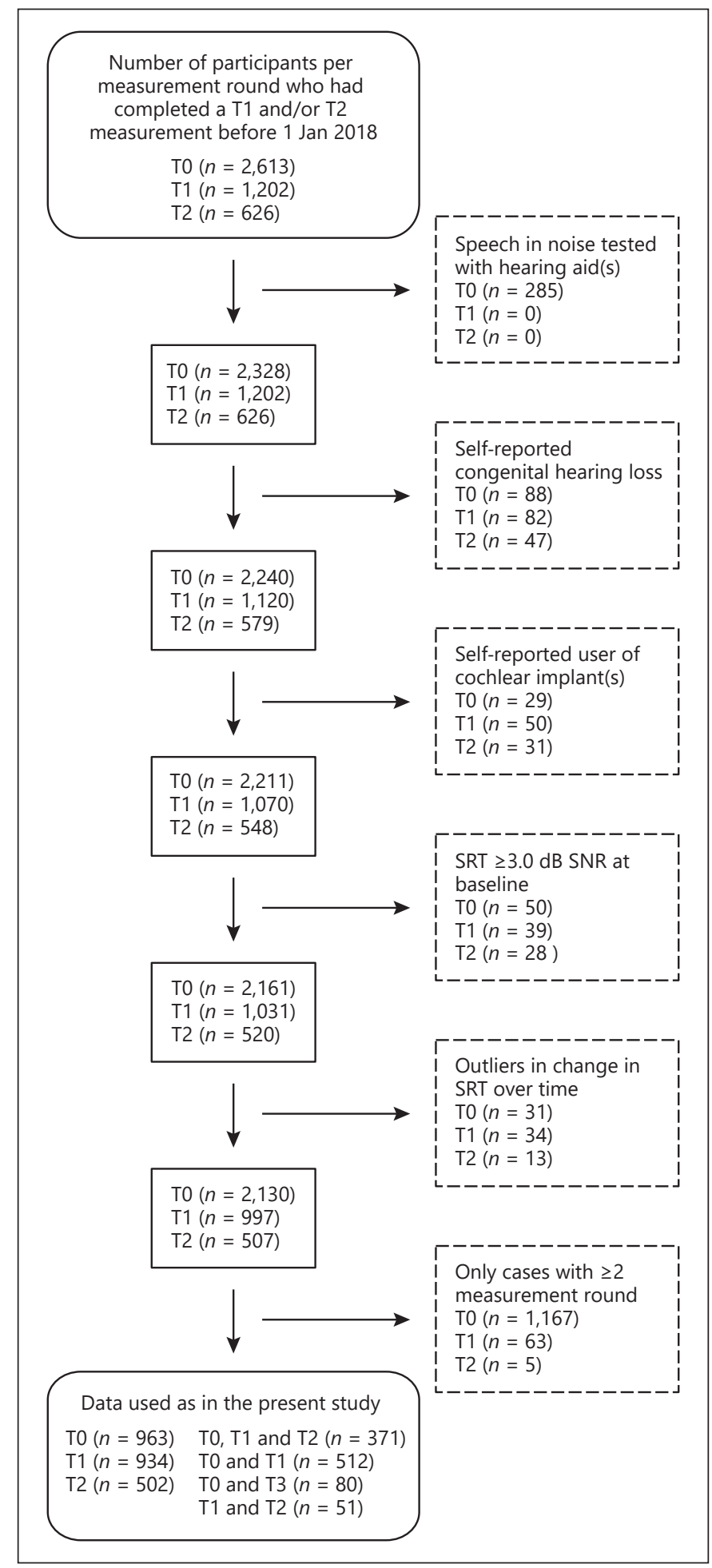

Fig. 1. Flowchart of participant numbers after applying inclusion criteria per measurement round. The number of measurements excluded per measurement round is given in the dashed line rectangle. SNR, signal-to-noise ratio; SRT, speech reception threshold; T0, baseline measurement; T1, 5-year follow-up measurement; T2, 10-year follow-up measurement.

Cardiovascular Disease Risk Factors and Speech Recognition in Noise
Table 1. Sample description

\begin{tabular}{llll}
\hline Variables & T0, & T1, & \multicolumn{1}{l}{ T2, } \\
& $N=963$ & $N=934$ & $N=502$ \\
\hline \multicolumn{2}{l}{$\begin{array}{l}\text { Demographic variables } \\
\text { Age group at baseline, } N(\%)\end{array}$} & \\
61-70 years & $121(12.6)$ & $120(12.8)$ & $49(9.8)$ \\
51-60 years & $329(34.2)$ & $320(34.3)$ & $164(32.7)$ \\
41-50 years & $243(25.2)$ & $232(24.8)$ & $152(30.3)$ \\
31-40 years & $146(15.2)$ & $145(15.5)$ & $83(16.5)$ \\
18-30 years & $124(12.9)$ & $117(12.5)$ & $54(10.8)$ \\
Sex, $N(\%)$ & & & \\
Female & $605(62.8)$ & $595(63.7)$ & $289(57.6)$ \\
Male & $358(37.2)$ & $339(36.3)$ & $213(42.4)$ \\
\hline
\end{tabular}

Hearing variables

Speech recognition ability in noise as indicated by the SRT, $N$ (\%)

$\begin{array}{llll}\text { Good } & 602(62.5) & 529(56.6) & 273(54.4) \\ \text { Insufficient } & 225(23.4) & 223(23.9) & 100(19.9) \\ \text { Poor } & 136(14.1) & 182(19.5) & 129(25.7)\end{array}$

Self-reported hearing (1-9 Likert scale; $1=$ very poor and $9=$ very good)

$\begin{array}{lccc}\text { 25th percentile } & 5 & 5 & 5 \\ \text { Median } & 6 & 7 & 6 \\ \text { 75th percentile } & 7 & 7 & 7 \\ \text { Transducer, } N(\%) & & & \\ \quad \text { Speaker } & 543(56.4) & 512(54.8) & 293(58.4) \\ \quad \text { Headphone } & 420(43.6) & 422(45.2) & 209(41.6)\end{array}$

Risk factors for CVD

Smoking, $N(\%)$

History of smoking $\quad 563(58.5) \quad 540(57.8) \quad 282(56.2)$

$\begin{array}{llll}\text { Never smoked } & 400(41.5) & 394(42.2) & 220(43.8)\end{array}$

Hypertension, $N(\%)$

Yes

$168(17.4) \quad 213(22.8) \quad 133(26.5)$

No

$792(82.2) \quad 721(77.2)$

$369(73.5)$

Missing

$3(0.3)$

0

0

Diabetes mellitus, $N(\%)$

Yes

$29(3.0) \quad 38(4.1) \quad 25(5.0)$

No

$931(96.7)$

$896(95.9)$

477 (95.0)

Missing

$3(0.3)$

0

0

Dyslipidemia, $N(\%)$

Yes

$53(5.5) \quad 75(8.0) \quad 54(10.8)$

No

$907(94.2)$

$448(89.2)$

Missing

$3(0.3)$

RA, $N(\%)$

Yes

No

$50(5.2)$

0

0

Missing

Obesity, $N(\%)$

Yes

No

Missing

$903(93.8)$

$867(92.8)$

$470(93.6)$

$10(1.0)$

$2(0.2)$

0

$80(8.3) \quad 81(8.7)$

$81(16.1)$

$433(45.0) \quad 403(43.1)$

$421(83.9)$

$450(46.7)$

$450(48.2)$

0

Cell entries display numbers of participants, with percentages in parentheses. T0, baseline measurement; T1, 5-year follow-up measurement; T2, 10-year follow-up measurement; SRT, speech reception threshold; CVD, cardiovascular disease; RA, rheumatoid arthritis. 
model. Variables considered as potential confounders were all included: CVD risk factors, age group, history of smoking, sex, and their interaction with time. In this model, the baseline age group was used as a time-independent variable. Note that analysis of the effect of obesity on SRT using the linear mixed model involved only participants who had a T2 measurement (besides a T0 or T1 measurement), as BMI data were only available at T2.

\section{Statistical Analysis: Main Effects of Risk Factors}

To analyze the main effects, another base model was built incorporating a random intercept for each participant and transducer type (headphones or loudspeakers) (i.e., base model $=$ random intercept + transducer type). First, the association between SRTs and single CVD risk factors was analyzed in the base model. Subsequently, risk factors were combined to analyze the possibility of a 2-way interaction (e.g., base model + hypertension + DM + hypertension $\times$ DM). In addition, sex, history of smoking, and age group were analyzed in the 2-way interactions. EMMs were calculated. Only interactions with a minimum of 15 participants per subgroup were analyzed.

All analyses were performed with and without adjustment for confounders. All CVD risk factors, age group, history of smoking, and sex were considered as potential confounders. In this model, the age group was used as a time-dependent variable. All statistical analyses were performed using SPSS version 26.0 (IBM Corp., Armonk, NY, USA). $p$ values $<0.05$ were considered indicating statistical significance.

\section{Results}

\section{Participants}

After applying the exclusion criteria, data from 1,014 out of a total of 2,613 participants were included and analyzed (Fig. 1). Mean age at baseline was 47.1 years $(\mathrm{SD}=$ 12.2 ), and $62.8 \%$ were female. Table 1 displays all relevant characteristics of the study population.

\section{Base Model}

The likelihood ratio test showed that adding a random slope did not improve the base model $\left(\chi^{2}[1]=0, p=1.0\right)$. This demonstrates that the variance in the rate of change of the SRT over time between participants was explained by natural variation and needed no correction. Tests performed with headphones yielded a better SRT than tests performed with loudspeakers $(F[1,2,026]=222.20, p<$ $0.001)$. The SRT decreased significantly over time $(F[2$, $1,441]=36.35, p<0.001$ ).

\section{Interactions between Risk Factors of CVD and Time}

None of the cardiovascular risk factors modified the decline in SRT over time significantly (Table 2). The only significant 3-way interaction observed was the interaction "RA $\times \operatorname{sex} \times$ time" $(F[2,1,557],=3.72, p=0.024)$.
Table 2. $F$ test for fixed effects

\begin{tabular}{|c|c|c|c|c|}
\hline & \multicolumn{4}{|c|}{ CVD risk factors; interaction with time } \\
\hline & $\begin{array}{l}\text { numerator } \\
\mathrm{dF}\end{array}$ & $\begin{array}{l}\text { denominator } \\
\mathrm{dF}\end{array}$ & $F$ & sig. \\
\hline Hypertension & 2 & 1,515 & 0.43 & 0.65 \\
\hline Hypertention $^{1}$ & 2 & 1,524 & 2.13 & 0.78 \\
\hline Diabetes & 2 & 1,532 & 0.47 & 0.62 \\
\hline Diabetes $^{1}$ & 2 & 1,537 & 1.01 & 0.37 \\
\hline Dyslipidemia & 2 & 1,549 & 0.06 & 0.94 \\
\hline Dyslipidemia $^{1}$ & 2 & 1,561 & 1.02 & 0.36 \\
\hline $\mathrm{RA}$ & 2 & 1,538 & 0.89 & 0.41 \\
\hline $\mathrm{RA}^{1}$ & 2 & 1,545 & 1.20 & 0.30 \\
\hline Obesity $^{2}$ & 2 & 944 & 0.24 & 0.79 \\
\hline
\end{tabular}

Unadjusted and adjusted models of the interaction between measurement rounds and CVD risk factors on SRT are given. $\mathrm{dF}$, degrees of freedom; $F, F$ value; sig, $p$ value; CVD, cardiovascular disease; RA, rheumatoid arthritis; SRT, speech reception threshold. ${ }^{1}$ Model adjusted for the age group. ${ }^{2}$ No confounders.

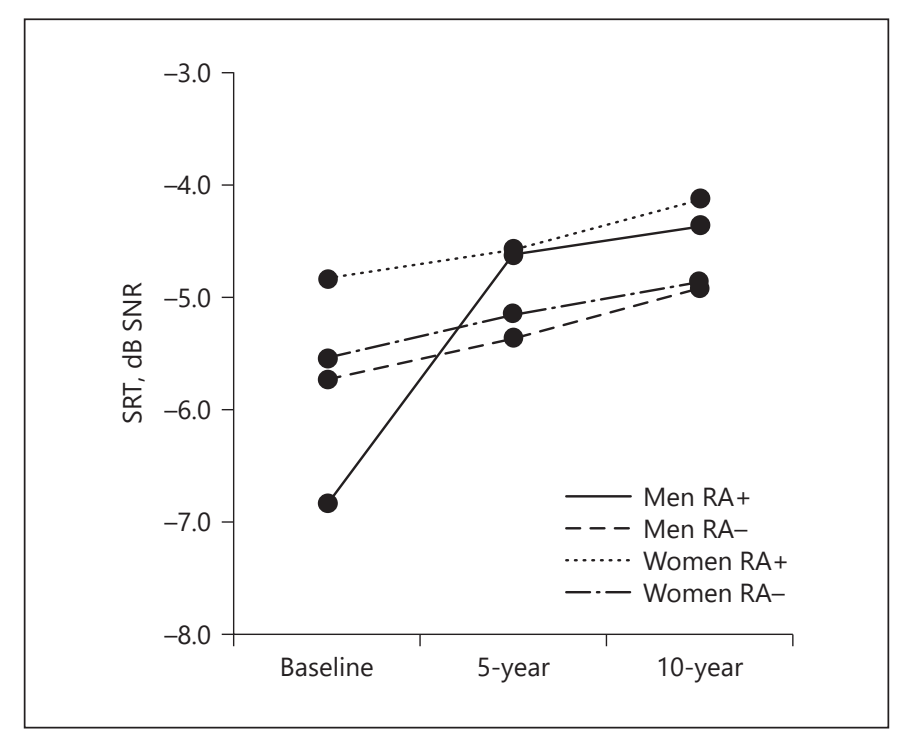

Fig. 2. EMMs of speech recognition in noise over 10 years stratified for RA and sex. EMMS, estimated marginal means; SRT, speech reception threshold; SNR, signal-to-noise ratio; RA, rheumatoid arthritis.

SRTs in male participants deteriorated faster in men with RA compared to men without RA while the decline was comparable in women with and without RA (Fig. 2, corresponding table with 95\% CIs is added as online suppl. Table 1; see www.karger.com/doi/10.1159/000513551 for all online suppl. material). The remaining 3-way interactions were not significant (online suppl. Table 2). 
Table 3. $F$ test for fixed effects

\begin{tabular}{|c|c|c|c|c|c|}
\hline & \multicolumn{5}{|c|}{ CVD risk factors; main effects } \\
\hline & $\begin{array}{l}\text { numerator } \\
\mathrm{dF}\end{array}$ & $\begin{array}{l}\text { denominator } \\
\mathrm{dF}\end{array}$ & $F$ & $\begin{array}{l}\text { regression coefficient } \\
\text { in } \mathrm{dB}(\mathrm{SNR}), 95 \% \mathrm{CI}\end{array}$ & sig. \\
\hline Hypertension & 1 & 2,310 & 13.63 & $0.49(0.23-0.75)$ & $<0.001$ \\
\hline Hypertention $^{1}$ & 1 & 2,232 & 4.06 & $0.27(0.01-0.53)$ & 0.044 \\
\hline Diabetes & 1 & 2,395 & 8.00 & $0.86(0.26-1.46)$ & 0.005 \\
\hline Diabetes $^{2}$ & 1 & 2,324 & 2.26 & $0.47(-0.14$ to 1.08$)$ & 0.13 \\
\hline Dyslipidemia & 1 & 2,108 & 7.82 & $0.52(0.16-0.89)$ & 0.005 \\
\hline Dyslipidemia $^{3}$ & 1 & 2,091 & 2.56 & $0.32(-0.07$ to 0.71$)$ & 0.11 \\
\hline RA & 1 & 2,084 & 7.25 & $0.55(0.15-0.94)$ & 0.007 \\
\hline $\mathrm{RA}^{3}$ & 1 & 2,012 & 4.89 & $0.45(0.05-0.86)$ & 0.027 \\
\hline Obesity $^{4}$ & 1 & 552 & 5.79 & $0.74(0.14-1.35)$ & 0.016 \\
\hline
\end{tabular}

Unadjusted and adjusted models of CVD risk factors on SRT are given. dF, degrees of freedom; $F, F$ value; sig, $p$ value; $C V D$, cardiovascular disease; SNR, signal-to-noise ratio; RA, rheumatoid arthritis; SRT, speech reception threshold. Statically significant $p$ values are printed in bold. ${ }^{1}$ Model adjusted for age group and sex. ${ }^{2}$ Model adjusted for age group, sex, and history of smoking. ${ }^{3}$ Model adjusted for age group. ${ }^{4}$ No confounders.

\section{Main Effects of Risk Factors of CVD}

Hypertension $(F[1,2,232]=4.06, p=0.044), \mathrm{RA}(F[1$, $2,012]=4.89, p=0.027)$, and obesity $(F[1,552]=5.79$, $p=0.016)$ significantly affected SRTs (Table 3 ). The estimated SRT of participants with hypertension was 0.27 dB SNR higher than the SRT of participants without hypertension (95\% CI: 0.01-0.53 dB SNR). Participants with RA had an estimated $0.45 \mathrm{~dB}$ SNR higher SRT compared to participants without RA (95\% CI: $0.05-0.86 \mathrm{~dB}$ SNR). Participants with obesity had an estimated 0.74 dB SNR (95\% CI: 0.14-1.35 dB SNR) higher SRT. None of the other variables were significantly associated with overall SRT (Table 3). Not significant but worth mentioning because of its effect size was the observed interaction between obesity and $\operatorname{DM}(F[1,1,437],=3.21, p=$ 0.073). In participants with DM, participants with obesity had a higher SRT $(\mathrm{EMM}=-3.99 \mathrm{~dB}$ SNR $[95 \% \mathrm{CI}=$ -5.21 to $-2.76 \mathrm{~dB}$ SNR]) compared to participants who were not obese $(\mathrm{EMM}=-6.04 \mathrm{~dB}$ SNR $[95 \% \mathrm{CI}=-7.06$ to $-5.03 \mathrm{~dB} \mathrm{SNR}]$ ), while in participants without DM, SRTs were only slightly higher in obese participants $(\mathrm{EMM}=-5.02 \mathrm{~dB}$ SNR $[95 \% \mathrm{CI}=-5.58$ to $-4.46 \mathrm{~dB}$ $\mathrm{SNR}]$ ) compared to nonobese participants (EMM = $-5.63 \mathrm{~dB}$ SNR [ $95 \% \mathrm{CI}=-5.88$ to $-5.37 \mathrm{~dB}$ SNR]). No significant 2-way interactions (i.e., between 2 CVD risk factors) were observed (online suppl. Table 3).

\section{Discussion}

In our previous study, we found that participants of the NL-SH with a history of tobacco smoking had a faster decline in SRT than participants without a history of tobacco smoking [Goderie et al., 2020]. As smoking is a risk factor for CVD, it raised the question if other longitudinal associations exist between hearing decline and CVD risk factors. We found no other longitudinal associations between hearing status (SRTs) and any of the CVD risk factors (hypertension, DM, RA, obesity, and dyslipidemia) over a 10 -year period. However, a longitudinal association of SRT with sex and RA was found. SRTs in male participants deteriorated faster in men with RA compared to men without RA. Females with RA did not differ from females without RA in the decline of SRTs over time. Overall participants with hypertension, RA, and obesity had higher (more unfavorable) SRTs. Among participants with DM, SRTs were higher in obese participants compared to nonobese participants (though not significant), while this difference was less pronounced in participants without DM.

Overall, RA was associated with higher (more unfavorable) SRTs, but no significant longitudinal association was observed. This confirms the results of Stam et al. [2014] using baseline NL-SH data only and those of other cross-sectional studies, showing that RA is associated with worse hearing [Ozturk et al., 2004; Takatsu et al., 2005; Stam et al., 2014; Jeong et al., 2016; Lee et al., 2019]. 
In contrast to our study, a study using reimbursement claims as an indicator of the presence of a chronic disease did show a longitudinal association between hearing loss and RA [Huang et al., 2018]. The different results could be explained by the self-reported information about RA in the current study, which might have led to misclassification, thereby obscuring a longitudinal relationship. The significant 3-way interaction between sex, RA, and time should be interpreted with caution because of the small sample size of men with RA and the baseline correction for SRT. Although the NL-SH cohort is of ample size, RA was reported in only 15, 24, and 15 men at T0, T1, and T2, respectively. This could have caused sampling error leading to an overestimation of the true change in SRT in men with RA. It was remarkable to observe that men with RA had a favorable average SRT at baseline and thereafter showed the largest decline in SRT. At follow-up, their SRT scores leveled with the average SRT of the other subgroups (i.e., men without RA and females). This could be interpreted as regression to the mean. Taking small sample size and regression to the mean into consideration, we argue that our significant finding of a larger decline in SRT in men with RA should be confirmed in other larger studies.

Although we found significant main effects of hypertension, RA, and obesity on SRTs, no interaction with time was found. A main effect represents a pooled analysis of longitudinal and cross-sectional effects. Cross-sectional effects are usually more susceptible for uncontrollable confounders and should be interpreted with caution. We therefore focused on longitudinal associations in this study which is a strength. If a strong association between a CVD risk factor and increased SRTs would exist, an interaction with time would be expected to appear. We failed to find a longitudinal association.

In participants with DM, obese participants had higher (more unfavorable) SRTs compared to nonobese participants with $\mathrm{DM}$, while in participants without DM, this difference was less pronounced. This association was not significant, but worth mentioning because of the large effect size. Note that the group having both DM and obesity was too small to include it in the longitudinal analysis. A meta-analysis of cross-sectional studies showed that $\mathrm{DM}$ is an independent risk factor for hearing loss [Horikawa et al., 2013]. However, longitudinal studies show conflicting results. Cruickshanks et al. [2015] found that poorly controlled DM and obesity led to an increased risk of developing hearing impairment, and obesity was associated with increased risk of developing self-reported hearing loss [Curhan et al., 2013]. Other studies did not reveal a longitudinal association between hearing loss and DM [Mitchell et al., 2009; Shargorodsky et al., 2010; Rigters et al., 2018]. The interaction between DM and obesity on speech recognition in noise to our knowledge has not been studied before.

Participants with hypertension had an overall worse SRT, but no longitudinal association between hearing decline and hypertension was found. Other longitudinal studies also failed to find a longitudinal association [Karlsmose et al., 2000; Shargorodsky et al., 2010; Cruickshanks et al., 2015]. However, some cross-sectional studies did report a significant association [Gates et al., 1993; Tan et al., 2018], indicating conflicting evidence. Of the traditional risk factors for CVD analyzed in this study, hypertension, RA, and obesity were significantly associated with higher (more unfavorable) SRTs overall, but no longitudinal associations between CVD risk factors and SRT were found. It is possible that longitudinal associations exist, but that these are weak or may have been obscured by other stronger risk factors, such as noise exposure and hereditary susceptibility. It is worthwhile to study these factors in future observational studies.

Nevertheless, the association between overall hypertension, obesity, and RA and higher SRTs observed in this study should be not ignored. In a previous study using the NL-SH cohort, a history of smoking and higher age (both CVD risk factors) were associated with a faster decline in SRTs [Goderie et al., 2020]. Altogether, these findings indicate that one should be alert about CVD factors possibly influencing someone's hearing status. In individuals with a hearing loss and CVD risk factors, we advise health care professionals to inform these individuals about the association between hearing loss and CVD risk factors. Health care professionals should be aware that hearing loss is associated with CVD risk factors when communicating with patients exposed to CVD risk factors, as hearing loss can play a significant role in the clinical encounter, hindering the communication with the health care professional [Mick et al., 2014; Reed et al., 2020].

\section{Strengths and Limitations}

This study holds several strengths. First, a large sample covering 5-10 years of follow-up was used. Second, by using longitudinal data, participants served as their own controls, thereby minimizing the effect of individual differences introducing selection bias as is often the case in cross-sectional studies. Third, a speech recognition in noise test was used. Decreased speech under- 
standing in noise was found to be the primary and most limiting consequence of hearing loss [Plomp and Mimpen, 1979; Kramer et al., 1998]. The SRT might therefore reflect a more ecologically valid assessment of functional hearing as compared to pure-tone audiometry thresholds. Last, we also studied the interaction of different CVD risk factors on change in SRT, next to the association between change of SRT with single CVD risk factors.

The diagnosis of dyslipidemia, hypertension, DM, and RA in this study was based on self-report and/or deducted from medication use (to reduce the effect of response bias) and not officially diagnosed by a doctor. This may be regarded as a limitation as it might have led to the following biases: (1) underreporting of disease because of undiagnosed diseases. (2) No differentiation between treated disease and untreated disease. (3) Antihypertensive medications are not always used for hypertension. (4) Under- or overreporting of diseases because of response bias. All these 4 points might have resulted in exposed participants being categorized in the unexposed group and vice versa. This may have attenuated a potentially existing association. If these biases had been random, the regression coefficient would have been attenuated and may have increased the likelihood of introducing type 2 errors [Hutcheon et al., 2010]. Regarding the effect of treated disease, it has been shown that patients treated for DM, hypertension, and dyslipidemia are still at increased risk for developing CVD, and thus even when treated, the presence of DM, hypertension, and dyslipidemia can still be regarded as CVD risk factors [D’Agostino et al., 2008; Leon and Maddox, 2015; Lieb et al., 2018].

Length of exposure to CVD risk factors is not included in the questionnaires, but it might affect hearing ability if an association exists between a CVD risk factor and hearing ability. However, the analyzed risk factors can be considered chronic and, therefore, are expected to be fairly stable over time.

When analyzing interactions between risk factors, sample sizes of subgroups of participants exposed to 2 risk factors became small. Small sample sizes of exposed participants to 1 or 2 CVD risk factors may have prevented us from detecting true associations.

The known measurement error of the NHT also needs to be considered (i.e., approximately $1 \mathrm{~dB}$ SNR) [Smits and Houtgast, 2005]. The relatively large measurement error reduces the sensitivity of the NHT test. Another limitation is the online data collection. Although convenient and relatively low cost, it does not allow for optimal standardization of the test condition and administration. This may imply that sometimes the NHT was done under suboptimal conditions, such as with poor-quality transducers or in a noisy environment. But, because of the nature of the test (i.e., speechin-noise test), the test result is relatively insensitive to ambient sound levels and type of equipment. We also attempted to control for these factors by giving explicit instructions and by controlling for transducer type in the statistical analysis.

More women than men take part in the NL-SH, and participants are on average more highly educated compared to the general population [Goderie et al., 2020]. However, compared to traditional methods, internet sampling has shown to increase geographic and demographic reach and reaches more effectively participants who are healthy at baseline, thus creating a more diverse sample [Gosling et al., 2004; Mathieu et al., 2013].

\section{Conclusion}

Obesity, RA, and hypertension were overall associated with higher SRTs. No significant interactions between 2 CVD risk factors and SRTs were found. Amongst the CVD risk factors investigated in this study, no CVD risk factors or interactions of 2 CVD risk factors were longitudinally associated with a higher decline in SRT. Health care professionals should be aware of the concurrent association between CVD risk factors and hearing loss.

\section{Acknowledgements}

The authors thank the participants of the Netherlands Longitudinal Study on Hearing (NL-SH).

\section{Statement of Ethics}

The NL-SH study protocol was approved by the Medical Ethics Committee of the Amsterdam UMC, Vrije Universiteit Amsterdam, Amsterdam, the Netherlands (2006/83; NL12015.029.06). Participants provided informed consent by actively enrolling in the study and filling out the baseline questionnaire.

\section{Conflict of Interest Statement}

The authors have no conflicts of interest to declare. 


\section{Funding Sources}

The first measurement round of the Netherlands Longitudinal Study on Hearing (NL-SH) (2006-2010) was financially supported by the Heinsius Houbolt Foundation, the Netherlands. Sonova AG, Switzerland, supported the data collection of the second measurement round (since 2011). Funding for data collection of the third measurement round (since 2016) came from the EMGO Institute for Health and Care Research, the Netherlands, and Sonova AG, Switzerland.

\section{Author Contributions}

T. Goderie, M. Stam, P. Merkus, C. Smits, and S.E. Kramer were involved in formulating the research questions and in designing the study. T. Goderie performed the analysis, and M. van Wier and B.I. Lissenberg-Witte verified the analytical methods. T. Goderie took the lead in writing the article. All authors provided critical feedback and helped shape the research, analysis, and article.

\section{References}

Agrawal Y, Platz EA, Niparko JK. Risk factors for hearing loss in US adults: data from the $\mathrm{Na}$ tional Health and Nutrition Examination Survey, 1999 to 2002. Otol Neurotol. 2009; 30(2):139-45

Anderson KM, Odell PM, Wilson PW, Kannel WB. Cardiovascular disease risk profiles. Am Heart J. 1991;121(1 Pt 2):293-8.

Besser J, Stropahl M, Urry E, Launer S. Comorbidities of hearing loss and the implications of multimorbidity for audiological care. Hear Res. 2018;369:3-14.

Bouwmans C, De Jong K, Timman R, ZijlstraVlasveld M, Van der Feltz-Cornelis C, Tan Swan S, et al. Feasibility, reliability and validity of a questionnaire on healthcare consumption and productivity loss in patients with a psychiatric disorder (TiC-P). BMC Health Serv Res. 2013;13:217.

Choy E, Ganeshalingam K, Semb AG, Szekanecz Z, Nurmohamed M. Cardiovascular risk in rheumatoid arthritis: recent advances in the understanding of the pivotal role of inflammation, risk predictors and the impact of treatment. Rheumatology. 2014;53(12):2143-54.

Cruickshanks KJ, Nondahl DM, Dalton DS, Fischer ME, Klein BE, Klein R, et al. Smoking, central adiposity, and poor glycemic control increase risk of hearing impairment. J Am Geriatr Soc. 2015;63(5):918-24.

Curhan SG, Eavey R, Wang M, Stampfer MJ, Curhan GC. Body mass index, waist circumference, physical activity, and risk of hearing loss in women. Am J Med. 2013;126(12): $1142-8$.

D'Agostino RB Sr, Vasan RS, Pencina MJ, Wolf PA, Cobain M, Massaro JM, et al. General cardiovascular risk profile for use in primary care: the Framingham Heart Study. Circulation. 2008;117:743-53.

Gates GA, Cobb JL, D’Agostino RB, Wolf PA. The relation of hearing in the elderly to the presence of cardiovascular disease and cardiovascular risk factors. Arch Otolaryngol Head Neck Surg. 1993;119(2):156-61.

Goderie TPM, Stam M, Lissenberg-Witte BI, Merkus P, Lemke U, Smits C, et al. 10-year follow-up results of The Netherlands Longitudinal Study on Hearing: trends of longitudinal change in speech recognition in noise. Ear Hear. 2020 May-Jun;41(3):491-9.
Gosling SD, Vazire S, Srivastava S, John OP. Should we trust web-based studies? A comparative analysis of six preconceptions about internet questionnaires. Am Psychol. 2004; 59(2):93-104

Guo Y, Zhang C, Du X, Nair U, Yoo TJ. Morphological and functional alterations of the cochlea in apolipoprotein $\mathrm{E}$ gene deficient mice. Hear Res. 2005;208(1-2):54-67.

Heo M, Faith MS, Pietrobelli A. Resistance to change of adulthood body mass index. Int J Obes Relat Metab Disord. 2002;26(10):14045.

Horikawa C, Kodama S, Tanaka S, Fujihara K, Hirasawa R, Yachi Y, et al. Diabetes and risk of hearing impairment in adults: a meta-analysis. J Clin Endocrinol Metab. 2013;98(1):518.

Huang CM, Chen HJ, Huang PH, Tsay GJ, Lan JL, Sung FC. Retrospective cohort study on risk of hearing loss in patients with rheumatoid arthritis using claims data. BMJ Open. 2018; 8(1):e018134.

Hutcheon JA, Chiolero A, Hanley JA. Random measurement error and regression dilution bias. BMJ. 2010;340:c2289.

Jeong H, Chang YS, Baek SY, Kim SW, Eun YH, Kim IY, et al. Evaluation of audiometric test results to determine hearing impairment in patients with rheumatoid arthritis: analysis of data from the Korean National Health and Nutrition Examination Survey. PLoS One. 2016;11(10):e0164591.

Karlsmose B, Lauritzen T, Engberg M, Parving A. A five-year longitudinal study of hearing in a Danish rural population aged $31-50$ years. $\mathrm{Br}$ J Audiol. 2000;34(1):47-55.

Katzmarzyk PT, Pérusse L, Malina RM, Bouchard C. Seven-year stability of indicators of obesity and adipose tissue distribution in the Canadian population. Am J Clin Nutr. 1999;69(6): 1123-9.

Kim JS, Lee H. Inner ear dysfunction due to vertebrobasilar ischemic stroke. Semin Neurol. 2009;29(5):534-40.

Kopelman PG. Obesity as a medical problem. Nature. 2000;404(6778):635-43.

Kramer SE, Kapteyn TS, Festen JM. The self-reported handicapping effect of hearing disabilities. Audiology. 1998;37(5):302-12.
Lee SY, Kong IG, Oh DJ, Choi HG. Increased risk of sudden sensory neural hearing loss in patients with rheumatoid arthritis: a longitudinal follow-up study using a national sample cohort. Clin Rheumatol. 2019;38(3):683-9.

Leon BM, Maddox TM. Diabetes and cardiovascular disease: epidemiology, biological mechanisms, treatment recommendations and future research. World J Diabetes. 2015;6(13): 1246-58.

Lieb W, Enserro DM, Larson MG, Vasan RS. Residual cardiovascular risk in individuals on lipid-lowering treatment: quantifying absolute and relative risk in the community. Open Heart. 2018;5(1):e000722.

Lin FR, Thorpe R, Gordon-Salant S, Ferrucci L. Hearing loss prevalence and risk factors among older adults in the United States. J Gerontol A Biol Sci Med Sci. 2011;66(5):58290.

Linssen AM, van Boxtel MP, Joore MA, Anteunis LJ. Predictors of hearing acuity: cross-sectional and longitudinal analysis. J Gerontol A Biol Sci Med Sci. 2014;69(6):759.

Lu Y, Hajifathalian K, Ezzati M, Woodward M, Rimm EB, Danaei G. Metabolic mediators of the effects of body-mass index, overweight, and obesity on coronary heart disease and stroke: a pooled analysis of 97 prospective cohorts with 1.8 million participants. Lancet. 2014;383:970-83.

Makishima K. Arteriolar sclerosis as a cause of presbycusis. Otolaryngology. 1978;86(2): Orl322-6.

Mathieu E, McGeechan K, Barratt A, Herbert R. Internet-based randomized controlled trials: a systematic review. J Am Med Inform Assoc. 2013;20(3):568-76.

Mick P, Foley DM, Lin FR. Hearing loss is associated with poorer ratings of patient-physician communication and healthcare quality. J Am Geriatr Soc. 2014;62(11):2207-9.

Mitchell P, Gopinath B, McMahon CM, Rochtchina E, Wang JJ, Boyages SC, et al. Relationship of type 2 diabetes to the prevalence, incidence and progression of age-related hearing loss. Diabet Med. 2009;26(5):483.

Mootz M, Van Den Berg J. Indicators of health status in the CBS-Health Interview Survey. Mndber Gezondheid. 1989;2:4-10. 
Morrell CH, Brant LJ, Ferrucci L. Model choice can obscure results in longitudinal studies. J Gerontol A Biol Sci Med Sci. 2009;64(2):21522.

Nomiya R, Nomiya S, Kariya S, Okano M, Morita $\mathrm{N}$, Cureoglu S, et al. Generalized arteriosclerosis and changes of the cochlea in young adults. Otol Neurotol. 2008;29(8):1193-7.

Oron Y, Elgart K, Marom T, Roth Y. Cardiovascular risk factors as causes for hearing impairment. Audiol Neurootol. 2014;19(4):256-60.

Ozturk A, Yalcin S, Kaygusuz I, Sahin S, Gok U, Karlidag T, et al. High-frequency hearing loss and middle ear involvement in rheumatoid arthritis. Am J Otolaryngol. 2004;25:411-7.

Plomp R, Mimpen AM. Speech-reception threshold for sentences as a function of age and noise level. J Acoust Soc Am. 1979;66(5): 1333-42.

Reed NS, Boss EF, Lin FR, Oh ES, Willink A. Satisfaction with quality of health care among Medicare beneficiaries with functional hearing loss. Med Care. 2020;59(1):22-8.
Rigters SC, van der Schroeff MP, Papageorgiou G, Baatenburg de Jong RJ, Goedegebure A. Progression of hearing loss in the aging population: repeated auditory measurements in the Rotterdam Study. Audiol Neurootol. 2018; 23(5):290-7.

Shargorodsky J, Curhan SG, Eavey R, Curhan GC. A prospective study of cardiovascular risk factors and incident hearing loss in men. Laryngoscope. 2010;120(9):1887-91.

Smits C, Houtgast T. Results from the Dutch speech-in-noise screening test by telephone. Ear Hear. 2005;26(1):89-95.

Smits C, Kapteyn TS, Houtgast T. Development and validation of an automatic speech-innoise screening test by telephone. Int J Audiol. 2004;43(1):15-28.

Smits C, Merkus P, Houtgast T. How we do it: the Dutch functional hearing-screening tests by telephone and internet. Clin Otolaryngol. 2006;31(5):436-40.

Stam M, Kostense PJ, Lemke U, Merkus P, Smit $\mathrm{JH}$, Festen JM, et al. Comorbidity in adults with hearing difficulties: Which chronic medical conditions are related to hearing impairment? Int J Audiol. 2014;53(6):392-401.
Stam M, Smits C, Twisk JW, Lemke U, Festen JM, Kramer SE. Deterioration of speech recognition ability over a period of 5 years in adults ages 18 to 70 years: results of the Dutch online speech-in-noise test. Ear Hear. 2015;36(3): e129-37.

Takatsu M, Higaki M, Kinoshita H, Mizushima Y, Koizuka I. Ear involvement in patients with rheumatoid arthritis. Otol Neurotol. 2005; 26(4):755-61.

Tan HE, Lan NSR, Knuiman MW, Divitini ML, Swanepoel DW, Hunter M, et al. Associations between cardiovascular disease and its risk factors with hearing loss-A cross-sectional analysis. Clin Otolaryngol. 2018;43(1):17281.

Vos T, Allen C, Arora M, Barber R, Bhutta Z, Brown AC, et al. Global, regional, and national incidence, prevalence, and years lived with disability for 310 diseases and injuries, 19902015: a systematic analysis for the Global Burden of Disease Study 2015. Lancet. 2016;388: 1545-602. 